Discrete Comput Geom 35:375-384 (2006)

DOI: $10.1007 / \mathrm{s} 00454-005-1201-3$

\title{
The Kissing Problem in Three Dimensions
}

\author{
Oleg R. Musin \\ Institute for the Mathematical Study of Complex Systems, \\ Moscow State University, \\ Moscow, Russia \\ omusin@mail.ru
}

\begin{abstract}
The kissing number $k(3)$ is the maximal number of equal size nonoverlapping spheres in three dimensions that can touch another sphere of the same size. This number was the subject of a famous discussion between Isaac Newton and David Gregory in 1694. The first proof that $k(3)=12$ was given by Schütte and van der Waerden only in 1953. In this paper we present a new solution of the Newton-Gregory problem that uses our extension of the Delsarte method. This proof relies on basic calculus and simple spherical geometry.
\end{abstract}

\section{Introduction}

The kissing number $k(d)$ is the highest number of equal nonoverlapping spheres in $\mathbf{R}^{d}$ that can touch another sphere of the same size. In three dimensions the kissing number problem is asking how many white billiard balls can kiss (touch) a black ball.

The most symmetrical configuration, 12 billiard balls around another, is achieved if the 12 balls are placed at positions corresponding to the vertices of a regular icosahedron concentric with the central ball. However, these 12 outer balls do not kiss each other and may all be moved freely. So perhaps if you moved all of them to one side a 13th ball would possibly fit in?

This problem was the subject of a famous discussion between Isaac Newton and David Gregory in 1694 (May 4, 1694; see the interesting article [21] for details of this discussion). Most reports say that Newton believed the answer was 12 balls, while Gregory thought that 13 might be possible. However, Casselman [5] found some puzzling features in this story.

This problem is often called the thirteen spheres problem. Hoppe [9] thought he had solved the problem in 1874 . However, there was a mistake-an analysis of this mistake was published by Hales in 1994 [8] (see also [20]). Finally, this problem was solved by Schütte and van der Waerden in 1953 [19]. A subsequent two-page sketch of an elegant 
proof was given by Leech [11] in 1956. Most people agree that Leech's proof is correct, but there are gaps in his exposition, many involving sophisticated spherical trigonometry. (Leech's proof was presented in the first edition of the well-known book by Aigner and Ziegler [1], the authors removed this chapter from the second edition because a complete proof would have had to include so much spherical trigonometry.) The thirteen spheres problem continues to be of interest, and new proofs have been published in the last few years by Hsiang [10], Maehara [13], Böröczky [3] and Anstreicher [2].

The main progress in the kissing number problem in high dimensions was at the end of the 1970s. Levenshtein [12] and, independently, Odlyzko and Sloane [16] $(=[6$, Chapter 13]) using Delsarte's method in 1979 proved that $k(8)=240$ and $k(24)=$ 196,560 . This proof is surprisingly short, clean, and technically easier than all proofs in three dimensions. However, $d=8,24$ are the only dimensions in which this method gives a precise result. For other cases (for instance, $d=3,4$ ) the upper bounds exceed the lower.

We found an extension of the Delsarte method in 2003 [14] (see details in [15]) that allowed us to prove the bound $k(4)<25$, i.e. $k(4)=24$. This extension also yields a proof $k(3)<13$.

The first version of these proofs was relatively short, but used a numerical solution of some nonconvex optimization problems. Later [15] these calculations were reduced to calculations of roots of polynomials in one variable.

In this paper we present a new proof of the Newton-Gregory problem. This proof needs just basic calculus and simple spherical geometry.

\section{2. $k(3)=12$}

We recall the definition of Legendre polynomials $P_{k}(t)$ by the recurrence formula:

$$
P_{0}=1, \quad P_{1}=t, \quad P_{2}=\frac{3}{2} t^{2}-\frac{1}{2}, \quad \ldots, \quad P_{k}=\frac{2 k-1}{k} t P_{k-1}-\frac{k-1}{k} P_{k-2}
$$

or equivalently

$$
P_{k}(t)=\frac{1}{2^{k} k !} \frac{d^{k}}{d t^{k}}\left(t^{2}-1\right)^{k} \quad \text { (Rodrigues' formula). }
$$

Lemma 1. Let $X=\left\{x_{1}, x_{2}, \ldots, x_{n}\right\}$ be any finite subset of the unit sphere $\mathbf{S}^{2}$ in $\mathbf{R}^{3}$. By $\phi_{i, j}=\operatorname{dist}\left(x_{i}, x_{j}\right)$ we denote the spherical (angular) distance between $x_{i}$ and $x_{j}$. Then

$$
\sum_{i=1}^{n} \sum_{j=1}^{n} P_{k}\left(\cos \left(\phi_{i, j}\right)\right) \geq 0 .
$$

This lemma easily follows from Schoenberg's theorem [18] for Gegenbauer (ultraspherical) polynomials $G_{k}^{(d)}$. (Note that $P_{k}=G_{k}^{(3)}$.) For completeness we give a proof of Lemma 1 in the Appendix.

Let

$$
f(t)=\frac{2431}{80} t^{9}-\frac{1287}{20} t^{7}+\frac{18333}{400} t^{5}+\frac{343}{40} t^{4}-\frac{83}{10} t^{3}-\frac{213}{100} t^{2}+\frac{t}{10}-\frac{1}{200} .
$$


Remark. This polynomial of degree 9 satisfies the assumptions of the extended Delsarte's method [14], [15]. An algorithm for calculating suitable polynomials is presented in the Appendix of [15].

Lemma 2. Suppose $X=\left\{x_{1}, x_{2}, \ldots, x_{n}\right\} \subset \mathbf{S}^{2}$. Then

$$
S(X)=\sum_{i=1}^{n} \sum_{j=1}^{n} f\left(\cos \left(\phi_{i, j}\right)\right) \geq n^{2} .
$$

Proof. The expansion of $f$ in terms of $P_{k}$ is

$$
f=\sum_{k=0}^{9} c_{k} P_{k}=P_{0}+\frac{8}{5} P_{1}+\frac{87}{25} P_{2}+\frac{33}{20} P_{3}+\frac{49}{25} P_{4}+\frac{1}{10} P_{5}+\frac{8}{25} P_{9} .
$$

We have $c_{0}=1, c_{k} \geq 0, k=1,2, \ldots, 9$. Using Lemma 1 we get

$$
S(X)=\sum_{k=0}^{9} c_{k} \sum_{i=1}^{n} \sum_{j=1}^{n} P_{k}\left(\cos \left(\phi_{i, j}\right)\right) \geq \sum_{i=1}^{n} \sum_{j=1}^{n} c_{0} P_{0}=n^{2} .
$$

If $n$ unit spheres kiss the unit sphere in $\mathbf{R}^{3}$, then the set of kissing points is an arrangement on the central sphere such that the (Euclidean) distance between any two points is at least 1 . So the kissing number problem can be stated in another way: How many points can be placed on the surface of $\mathbf{S}^{2}$ so that the angular separation between any two points is at least $60^{\circ}$ ?

Lemma 3. Suppose $X=\left\{x_{1}, x_{2}, \ldots, x_{n}\right\}$ is a subset of $\mathbf{S}^{2}$ such that the angular separation $\phi_{i, j}$ between any two distinct points $x_{i}, x_{j}$ is at least $60^{\circ}$. Then

$$
S(X)=\sum_{i=1}^{n} \sum_{j=1}^{n} f\left(\cos \left(\phi_{i, j}\right)\right)<13 n .
$$

We give a proof of Lemma 3 in the next section.

Theorem. $k(3)=12$.

Proof. Suppose $X$ is a kissing arrangement on $\mathbf{S}^{2}$ with $n=k(3)$. Then $X$ satisfies the assumptions in Lemmas 2 and 3. Therefore, $n^{2} \leq S(X)<13 n$. From this $n<13$ follows, i.e. $n \leq 12$. From the other side we have $k(3) \geq 12$, showing that $n=k(3)=12$.

\section{Proof of Lemma 3}

We need one fact from spherical trigonometry, namely the law of cosines:

$\cos \phi=\cos \theta_{1} \cos \theta_{2}+\sin \theta_{1} \sin \theta_{2} \cos \varphi$, 


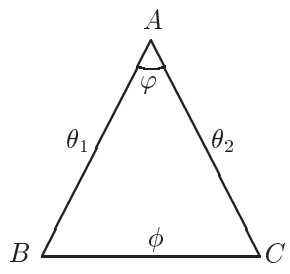

Fig. 1

for a spherical triangle $A B C$ with sides of angular lengths $\theta_{1}, \theta_{2}, \phi$ and $\angle B A C=\varphi$ (Fig. 1). For $\varphi=90^{\circ}$, this reduces to the spherical Pythagorean theorem: $\cos \phi=\cos \theta_{1} \cos \theta_{2}$.

Proof. 1. The polynomial $f(t)$ satisfies the following properties (see Fig. 2):

(i) $f(t)$ is a monotone decreasing function on the interval $\left[-1,-t_{0}\right]$;

(ii) $f(t)<0$ for $t \in\left(-t_{0}, \frac{1}{2}\right]$;

where $f\left(-t_{0}\right)=0, t_{0} \approx 0.5907$.

These properties hold because $f(t)$ has only one root $-t_{0}$ on $\left[-1, \frac{1}{2}\right]$, and there are no zeros of the derivative $f^{\prime}(t)$ (eighth degree polynomial) on $\left[-1,-t_{0}\right]$.

Let $S_{i}(X):=\sum_{j=1}^{n} f\left(\cos \left(\phi_{i, j}\right)\right)$, then $S(X)=\sum_{i=1}^{n} S_{i}(X)$. From this it follows that if $S_{i}(X)<13$ for $i=1,2, \ldots, n$, then $S(X)<13 n$.

We obviously have $\phi_{i, i}=0$, so $f\left(\cos \phi_{i, i}\right)=f(1)$. Note that our assumption on $X$ $\left(\phi_{i, j} \geq 60^{\circ}, i \neq j\right)$ yields $\cos \phi_{i, j} \leq \frac{1}{2}$. Therefore, $\cos \phi_{i, j}$ lies in the interval $\left[-1, \frac{1}{2}\right]$. By (ii) we have $f\left(\cos \phi_{i, j}\right) \leq 0$ whenever $\cos \phi_{i, j} \in\left[-t_{0}, \frac{1}{2}\right]$. Let $J(i):=\left\{j: \cos \phi_{i, j} \in\right.$ $\left.\left[-1,-t_{0}\right)\right\}$. We obtain

$$
S_{i}(X) \leq T_{i}(X):=f(1)+\sum_{j \in J(i)} f\left(\cos \phi_{i, j}\right) .
$$

Let $\theta_{0}=\arccos t_{0} \approx 53.794^{\circ}$. Then $j \in J(i)$ iff $\phi_{i, j}>180^{\circ}-\theta_{0}$, i.e. $\theta_{j}<\theta_{0}$, where $\theta_{j}=180^{\circ}-\phi_{i, j}$. In other words all $x_{i, j}, j \in J(i)$, lie inside the spherical cap of center $e_{0}$ and radius $\theta_{0}$, where $e_{0}=-x_{i}$ is the antipodal point to $x_{i}$.

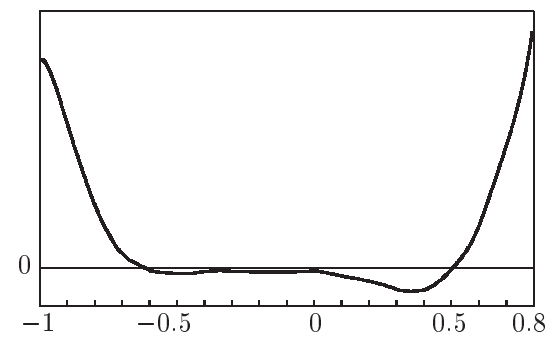

Fig. 2. The graph of the function $f(t)$ 
2. Let us consider on $\mathbf{S}^{2}$ points $e_{0}, y_{1}, \ldots, y_{m}$ such that

$$
\begin{gathered}
\phi_{i, j}=\operatorname{dist}\left(y_{i}, y_{j}\right) \geq 60^{\circ}, \quad \forall i \neq j, \\
\theta_{i}:=\operatorname{dist}\left(e_{0}, y_{i}\right)<\theta_{0} \quad \text { for } \quad 1 \leq i \leq m .
\end{gathered}
$$

Denote by $\mu$ the highest value of $m$ such that the constraints in (2) allow a nonempty set of points $y_{1}, \ldots, y_{m}$.

Suppose that $0 \leq m \leq \mu$ and $Y=\left\{y_{1}, \ldots, y_{m}\right\}$ satisfies (2). Let

$$
\begin{gathered}
H(Y)=H\left(y_{1}, \ldots, y_{m}\right):=f(1)+f\left(-\cos \theta_{1}\right)+\cdots+f\left(-\cos \theta_{m}\right), \\
h_{m}:=\sup _{Y}\{H(Y)\}, \quad h_{\max }:=\max \left\{h_{0}, h_{1}, \ldots, h_{\mu}\right\} .
\end{gathered}
$$

It is clear that $T_{i}(X) \leq h_{m}$, where $m=|J(i)|$. From (1) it follows that $S_{i}(X) \leq h_{m}$. Thus, if we prove that $h_{\max }<13$, then we prove Lemma 3 .

3. Now we prove that $\mu \leq 4$.

Suppose $Y=\left\{y_{1}, \ldots, y_{m}\right\} \subset \mathbf{S}^{2}$ satisfies (2). By symmetry we may assume that $e_{0}$ is the North pole and $y_{i}$ has polar coordinates $\left(\theta_{i}, \varphi_{i}\right)$. Then from the law of cosines we have

$$
\cos \phi_{i, j}=\cos \theta_{i} \cos \theta_{j}+\sin \theta_{i} \sin \theta_{j} \cos \left(\varphi_{i}-\varphi_{j}\right) .
$$

Note that $\theta_{i}>0$ for $m \geq 2$. Conversely, $y_{i}=e_{0}, \theta_{j}=\phi_{i, j} \geq 60^{\circ}>\theta_{0}$, a contradiction. From (2) we have $\cos \phi_{i, j} \leq \frac{1}{2}$, then

$$
\cos \left(\varphi_{i}-\varphi_{j}\right) \leq \frac{\frac{1}{2}-\cos \theta_{i} \cos \theta_{j}}{\sin \theta_{i} \sin \theta_{j}}
$$

Let

$$
Q(\alpha, \beta)=\frac{\frac{1}{2}-\cos \alpha \cos \beta}{\sin \alpha \sin \beta},
$$

then

$$
Q_{\alpha}^{\prime}(\alpha, \beta)=\frac{\partial Q(\alpha, \beta)}{\partial \alpha}=\frac{2 \cos \beta-\cos \alpha}{2 \sin ^{2} \alpha \sin \beta} .
$$

From this it follows that if $0<\alpha, \beta \leq \theta_{0}$, then $\cos \beta>\frac{1}{2}$ (because $\theta_{0}<60^{\circ}$ ); so then $Q_{\alpha}^{\prime}(\alpha, \beta)>0$, and $Q(\alpha, \beta) \leq Q\left(\theta_{0}, \beta\right) \leq Q\left(\theta_{0}, \theta_{0}\right)$. Therefore,

$$
\frac{\frac{1}{2}-\cos \theta_{i} \cos \theta_{j}}{\sin \theta_{i} \sin \theta_{j}} \leq \frac{\frac{1}{2}-\cos ^{2} \theta_{0}}{\sin ^{2} \theta_{0}}=\frac{\frac{1}{2}-t_{0}^{2}}{1-t_{0}^{2}} .
$$

Combining this inequality and (3), we get

$$
\cos \left(\varphi_{i}-\varphi_{j}\right) \leq \frac{\frac{1}{2}-t_{0}^{2}}{1-t_{0}^{2}}
$$

Note that $\arccos \left(\left(\frac{1}{2}-t_{0}^{2}\right) /\left(1-t_{0}^{2}\right)\right) \approx 76.582^{\circ}>72^{\circ}$. This implies that $m \leq 4$ because no more than four points can lie in a circle with the minimum angular separation between any two points greater than $72^{\circ}$. 


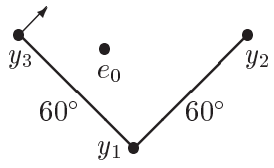

Fig. 3

4. Now we have to prove that $h_{\max }=\max \left\{h_{0}, h_{1}, h_{2}, h_{3}, h_{4}\right\}<13$. We obviously have $h_{0}=f(1)=10.11<13$.

From (i) follows that $f(-\cos \theta)$ is a monotone decreasing function in $\theta$ on $\left[0, \theta_{0}\right]$. Then, for $m=1, H\left(y_{1}\right)=f(1)+f\left(-\cos \theta_{1}\right)$ attains its maximum at $\theta_{1}=0$,

$$
h_{1}=f(1)+f(-1)=12.88<13 .
$$

5. Let us consider for $m=2,3,4$ an arrangement $\left\{e_{0}, y_{1}, \ldots, y_{m}\right\}$ in $\mathbf{S}^{2}$ that gives $H(Y)=h_{m}$. Here $y_{i} \neq e_{0}$ (see $\mathbf{3}$ ). Note that in this arrangement, points $y_{k}$ cannot be shifted towards $e_{0}$ because in this case $H(Y)$ increases.

For $m=2$ this yields $e_{0} \in y_{1} y_{2}$, and $\operatorname{dist}\left(y_{1}, y_{2}\right)=60^{\circ}$. If $e_{0} \notin y_{1} y_{2}$, then the whole arc $y_{1} y_{2}$ can be shifted towards $e_{0}$. If $\operatorname{dist}\left(y_{1}, y_{2}\right)>60^{\circ}$, then $y_{1}$ (and $\left.y_{2}\right)$ can be shifted towards $e_{0}$.

For $m=3$ we prove that $\Delta_{3}=y_{1} y_{2} y_{3}$ is a spherical regular triangle with edge length $60^{\circ}$. As above, $e_{0} \in \Delta_{3}$, otherwise the whole triangle can be shifted towards $e_{0}$. Suppose $\operatorname{dist}\left(y_{1}, y_{i}\right)>60^{\circ}, i=2,3$, then $\operatorname{dist}\left(y_{1}, e_{0}\right)$ can be decreased. From this, it follows that for any $y_{i}$ at least one of the distances $\left\{\operatorname{dist}\left(y_{i}, y_{j}\right)\right\}$ is equal to $60^{\circ}$. Therefore, at least two sides of $\Delta_{3}$ (say $y_{1} y_{2}$ and $y_{1} y_{3}$ ) have length $60^{\circ}$. Also dist $\left(y_{2}, y_{3}\right)=60^{\circ}$, conversely $y_{3}$ (or $y_{2}$ if $e_{0} \in y_{1} y_{3}$ ) can be rotated about $y_{1}$ by a small angle towards $e_{0}$ (Fig. 3).

For $m=4$ we first prove that $\Delta_{4}:=\operatorname{conv} Y$ (the spherical convex hull of $Y$ ) is a convex quadrilateral. Conversely, we may assume that $y_{4} \in y_{1} y_{2} y_{3}$.

The great circle through $y_{4}$ that is orthogonal to the arc $e_{0} y_{4}$ divides $\mathbf{S}^{2}$ into two hemispheres: $H_{1}$ and $H_{2}$. Suppose $e_{0} \in H_{1}$, then at least one $y_{i}$ (say $y_{3}$ ) belongs to $H_{2}$ (Fig. 4). So the angle $\angle e_{0} y_{4} y_{3}$ is greater than $90^{\circ}$, then (again from the law of cosines $) \operatorname{dist}\left(y_{3}, e_{0}\right)>\operatorname{dist}\left(y_{3}, y_{4}\right)$. Thus, $\theta_{3}=\operatorname{dist}\left(y_{3}, e_{0}\right)>\operatorname{dist}\left(y_{3}, y_{4}\right) \geq 60^{\circ}>\theta_{0}$, a contradiction.

Arguing as for $m=3$ it is easy to prove that for any vertex $y_{i}$ there are at least two vertices $y_{j}$ at a distance $60^{\circ}$ from $y_{i}$. Note that the diagonals of $\Delta_{4}$ cannot both be of length $60^{\circ}$. Conversely, at least one side of $\Delta_{4}$ is of length less than $60^{\circ}$. Thus, $\Delta_{4}$ is a spherical equilateral quadrangle (rhomb) with edge length $60^{\circ}$.

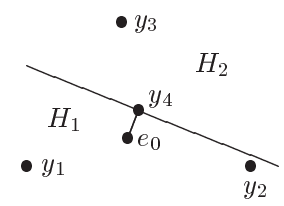

Fig. 4 
6. Now we introduce the function $F_{1}(\psi),{ }^{1}$ where $\psi \in\left[60^{\circ}, 2 \theta_{0}\right]$ :

$$
F_{1}(\psi):=\max _{\psi / 2 \leq \theta \leq \theta_{0}}\left\{\widetilde{F}_{1}(\theta, \psi)\right\}, \quad \widetilde{F}_{1}(\theta, \psi)=f(-\cos \theta)+f(-\cos (\psi-\theta)) .
$$

So if $\operatorname{dist}\left(y_{i}, y_{j}\right)=\psi$, then

$$
f\left(-\cos \theta_{i}\right)+f\left(-\cos \theta_{j}\right) \leq F_{1}(\psi)
$$

Therefore,

$$
H\left(y_{1}, y_{2}\right) \leq h_{2}=f(1)+F_{1}\left(60^{\circ}\right) \approx 12.8749<13 .
$$

7. Let $m=4, d_{1}=\operatorname{dist}\left(y_{1}, y_{3}\right)$ and $d_{2}=\operatorname{dist}\left(y_{2}, y_{4}\right)$. Since $\Delta_{4}=y_{1} y_{2} y_{3} y_{4}$ is a spherical rhomb, we have $\cos \left(d_{1} / 2\right) \cos \left(d_{2} / 2\right)=\frac{1}{2}$ (Pythagorean theorem, the diagonals $y_{1} y_{3}, y_{2} y_{4}$ of $\Delta_{4}$ are orthogonal). So if

$$
\rho(s):=2 \arccos \frac{1}{2 \cos (s / 2)},
$$

then

$$
\rho\left(d_{1}\right)=d_{2}, \quad \rho\left(d_{2}\right)=d_{1}, \quad \rho\left(90^{\circ}\right)=90^{\circ}, \quad \rho(\rho(s))=s .
$$

Suppose $d_{1} \leq d_{2}$. The inequalities $\theta_{i} \leq \theta_{0}$ yield $d_{2} \leq 2 \theta_{0}$. Then

$$
\rho\left(2 \theta_{0}\right) \leq d_{1} \leq 90^{\circ} \leq d_{2} \leq 2 \theta_{0}
$$

Now we consider two cases:

(1) $\rho\left(2 \theta_{0}\right) \leq d_{1}<77^{\circ}$, and

(2) $77^{\circ} \leq d_{1} \leq 90^{\circ}$.

(1) Clearly, $F_{1}(\psi)$ is a monotone decreasing function in $\psi$. Then (4) implies

$$
\begin{gathered}
f\left(-\cos \theta_{1}\right)+f\left(-\cos \theta_{3}\right) \leq F_{1}\left(d_{1}\right) \leq F_{1}\left(\rho\left(2 \theta_{0}\right)\right), \\
f\left(-\cos \theta_{2}\right)+f\left(-\cos \theta_{4}\right) \leq F_{1}\left(d_{2}\right)=F_{1}\left(\rho\left(d_{1}\right)\right)<F_{1}\left(\rho\left(77^{\circ}\right)\right),
\end{gathered}
$$

so then

$$
H(Y)<f(1)+F_{1}\left(\rho\left(2 \theta_{0}\right)\right)+F_{1}\left(\rho\left(77^{\circ}\right)\right) \approx 12.9171<13 .
$$

(2) In this case we have

$$
H(Y) \leq f(1)+F_{1}\left(77^{\circ}\right)+F_{1}\left(90^{\circ}\right) \approx 12.9182<13
$$

Thus, $h_{4}<13$.

\footnotetext{
${ }^{1}$ For given $\psi$, the value $F_{1}(\psi)$ can be found as the maximum of the ninth degree polynomial $\Omega(s)=$ $\widetilde{F}_{1}(\theta, \psi), s=\cos (\theta-\psi / 2)$, on the interval $\left[\cos \left(\theta_{0}-\psi / 2\right), 1\right]$.
} 


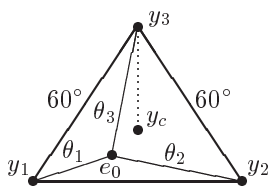

Fig. 5

8. Our last step is to show that $h_{3}<13$. $^{2}$

Since $\Delta_{3}$ is a regular triangle, $H(Y)=f(1)+f\left(-\cos \theta_{1}\right)+f\left(-\cos \theta_{2}\right)+f\left(-\cos \theta_{3}\right)$ is a symmetric function in the $\theta_{i}$, so it is sufficient to consider the case $\theta_{1} \leq \theta_{2} \leq \theta_{3} \leq \theta_{0}$. In this case $R_{0} \leq \theta_{3} \leq \theta_{0}$, where $R_{0}=\arccos \sqrt{2 / 3} \approx 35.2644^{\circ}$ is the (spherical) circumradius of $\Delta_{3}$.

Let $y_{c}$ be the center of $\Delta_{3}$. We have $\gamma:=\angle y_{1} y_{3} y_{c}=\angle y_{2} y_{3} y_{c}$. Using the law of cosines for the triangle $y_{1} y_{3} y_{c}$, we get $\gamma=\arccos \sqrt{2 / 3}$, i.e. $\gamma=R_{0}$.

Denote the angle $\angle e_{0} y_{3} y_{c}$ by $u$. Then (see Fig. 5)

$$
\begin{aligned}
& \cos \theta_{1}=\cos 60^{\circ} \cos \theta_{3}+\sin 60^{\circ} \sin \theta_{3} \cos \left(R_{0}-u\right), \\
& \cos \theta_{2}=\cos 60^{\circ} \cos \theta_{3}+\sin 60^{\circ} \sin \theta_{3} \cos \left(R_{0}+u\right),
\end{aligned}
$$

where $0 \leq u \leq u_{0}:=\arccos \left(\cot \theta_{3} / \sqrt{3}\right)-R_{0}$. Note that if $u=u_{0}$, then $\theta_{2}=\theta_{3} ; u=0$ yields $\theta_{1}=\theta_{2}$; and if $0<u<u_{0}$, then $\theta_{1}<\theta_{2}<\theta_{3}$.

For fixed $\theta_{3}=\psi, H\left(y_{1}, y_{2}\right)$ is a polynomial of degree 9 in $s=\cos u$. Denote by $F_{2}(\psi)$ the maximum of this polynomial on the interval $\left[\cos u_{0}, 1\right]$.

Let

$$
\left\{\psi_{1}, \ldots, \psi_{6}\right\}=\left\{R_{0}, 38^{\circ}, 41^{\circ}, 44^{\circ}, 48^{\circ}, \theta_{0}\right\} .
$$

It is clear that $F_{2}(\psi)$ is a monotone increasing function in $\psi$ on $\left[R_{0}, \theta_{0}\right]$. From the other side, $f(-\cos \psi)$ is a monotone decreasing function in $\psi$. Therefore for $\theta_{3} \in\left[\psi_{i}, \psi_{i+1}\right]$ we have

$$
H(Y)=H\left(y_{1}, y_{2}\right)+f\left(-\cos \theta_{3}\right)<w_{i}:=F_{2}\left(\psi_{i+1}\right)+f\left(-\cos \psi_{i}\right) .
$$

Since,

$$
\left\{w_{1}, \ldots, w_{5}\right\} \approx\{12.9425,12.9648,12.9508,12.9606,12.9519\},
$$

we get $h_{3}<\max \left\{w_{i}\right\}<13$.

Thus, $h_{m}<13$ for all $m$ as required.

\section{Appendix. Proof of Lemma 1.}

In this proof we use Schoenberg's original proof [18] which is based on the addition theorem for Gegenbauer polynomials. ${ }^{3}$ The addition theorem for Legendre polynomials

\footnotetext{
${ }^{2}$ A more detailed analysis shows $h_{3} \approx 12.8721, h_{4} \approx 12.4849$.

${ }^{3}$ Pfender and Ziegler [17] give a proof as a simple consequence of the addition theorem for spherical harmonics. This theorem is not so elementary. The addition theorem for Legendre polynomials can be proven by elementary algebraic calculations.
} 
was discovered by Laplace and Legendre in 1782-1785:

$$
\begin{aligned}
& P_{k}\left(\cos \theta_{1} \cos \theta_{2}+\sin \theta_{1} \sin \theta_{2} \cos \varphi\right) \\
& \quad=P_{k}\left(\cos \theta_{1}\right) P_{k}\left(\cos \theta_{2}\right)+2 \sum_{m=1}^{k} \frac{(k-m) !}{(k+m) !} P_{k}^{m}\left(\cos \theta_{1}\right) P_{k}^{m}\left(\cos \theta_{2}\right) \cos m \varphi \\
& \quad=\sum_{m=0}^{k} c_{m, k} P_{k}^{m}\left(\cos \theta_{1}\right) P_{k}^{m}\left(\cos \theta_{2}\right) \cos m \varphi,
\end{aligned}
$$

where

$$
P_{k}^{m}(t)=\left(1-t^{2}\right)^{m / 2} \frac{d^{m}}{d t^{m}} P_{k}(t) .
$$

(See the details in [4] and [7].)

Proof. Let $X=\left\{x_{1}, \ldots, x_{n}\right\} \subset \mathbf{S}^{2}$ and let $x_{i}$ have spherical (polar) coordinates $\left(\theta_{i}, \varphi_{i}\right)$. Then from the law of cosines we have

$$
\cos \phi_{i, j}=\cos \theta_{i} \cos \theta_{j}+\sin \theta_{i} \sin \theta_{j} \cos \varphi_{i, j}, \quad \varphi_{i, j}:=\varphi_{i}-\varphi_{j},
$$

which yields

$$
\begin{aligned}
\sum_{i, j} P_{k}\left(\cos \phi_{i, j}\right) & =\sum_{i, j} \sum_{m=0}^{k} c_{m, k} P_{k}^{m}\left(\cos \theta_{i}\right) P_{k}^{m}\left(\cos \theta_{j}\right) \cos m \varphi_{i, j} \\
& =\sum_{m} c_{m, k} \sum_{i, j} u_{m, i} u_{m, j} \cos m \varphi_{i, j}, \quad u_{m, i}=P_{k}^{m}\left(\cos \theta_{i}\right) .
\end{aligned}
$$

We prove that for any real $u_{1}, \ldots, u_{n}$,

$$
\sum_{i, j} u_{i} u_{j} \cos m \varphi_{i, j} \geq 0 .
$$

Pick $n$ vectors $v_{1}, \ldots, v_{n}$ in $\mathbf{R}^{2}$ with coordinates $v_{i}=\left(\cos m \varphi_{i}, \sin m \varphi_{i}\right)$. If $v=u_{1} v_{1}+$ $\cdots+u_{n} v_{n}$, then

$$
0 \leq\|v\|^{2}=\langle v, v\rangle=\sum_{i, j} u_{i} u_{j} \cos m \varphi_{i, j} .
$$

This inequality and the inequalities $c_{m, k}>0$ complete our proof.

\section{References}

1. M. Aigner and G.M. Ziegler, Proofs from THE BOOK, Springer, New York, 1998 (first edn.), 2002 (second edn.)

2. K. Anstreicher, The thirteen spheres: a new proof, Discrete Comput. Geom., 31 (2004), 613-625.

3. K. Böröczky, The Newton-Gregory problem revisited, Proc. Discrete Geometry, Marcel Dekker, New York, 2003, pp. 103-110.

4. B.C. Carlson, Special Functions of Applied Mathematics, Academic Press, New York, 1977. 
5. B. Casselman, The difficulties of kissing in three dimensions, Notices Amer. Math. Soc. 51 (2004), 884885.

6. J.H. Conway and N.J.A. Sloane, Sphere Packings, Lattices, and Groups, Springer-Verlag, New York, 1999 (third edn.).

7. A. Erdélyi (editor), Higher Transcendental Function, McGraw-Hill, New York, 1953, Volume II, Chapter XI.

8. T. Hales, The status of the Kepler conjecture, Math. Intelligencer 16 (1994), 47-58.

9. R. Hoppe, Bemerkung der Redaction, Archiv Math. Phys. (Grunet) 56 (1874), 307-312.

10. W.-Y. Hsiang, Least Action Principle of Crystal Formation of Dense Packing Type and Kepler's Conjecture, World Scientific, Singapore, 2001.

11. J. Leech, The problem of the thirteen spheres, Math. Gaz. 41 (1956), 22-23.

12. V.I. Levenshtein, On bounds for packing in $n$-dimensional Euclidean space, Soviet Math. Dokl. 20(2), $1979,417-421$.

13. H. Maehara, Isoperimetric theorem for spherical polygons and the problem of 13 spheres, Ryukyu Math.J. 14 (2001), 41-57.

14. O.R. Musin, The problem of the twenty-five spheres, Russian Math. Surveys 58 (2003), 794-795.

15. O.R. Musin, The kissing number in four dimensions, Preprint, September 2003, math. MG/0309430.

16. A.M. Odlyzko and N.J.A. Sloane, New bounds on the number of unit spheres that can touch a unit sphere in $n$ dimensions, J. Combin. Theory Ser. A 26 (1979), 210-214.

17. F. Pfender and G.M. Ziegler, Kissing numbers, sphere packings, and some unexpected proofs, Notices Amer. Math. Soc. 51 (2004), 873-883.

18. I.J. Schoenberg, Positive definite functions on spheres, Duke Math. J. 9 (1942), 96-107.

19. K. Schütte and B.L. van der Waerden, Das Problem der dreizehn Kugeln, Math. Ann. 125 (1953), 325-334.

20. G.G. Szpiro, Kepler's Conjecture, Wiley, New York, 2002.

21. G.G. Szpiro, Newton and the kissing problem, http://plus.maths.org/issue23/features/kissing/

Received November 18, 2004, and in revised form June 11, 2005. Online publication October 25, 2005. 